\title{
Alternative models of familiarity and false claims concerning social recognition systems
}

\author{
Paulien J. A. de Bruijn • Maurice W. Sabelis • \\ Martijn Egas
}

Received: 7 August 2014 / Accepted: 14 August 2014/Published online: 23 August 2014

(C) Springer-Verlag Berlin Heidelberg 2014

Thrips represent an ideal biological system to investigate mechanisms of social recognition. In his commentary on our article (de Bruijn et al. 2014) introducing thrips for this purpose, Schausberger (2014) assumes a social recognition system in which individuals should memorize 'distinct individual labels of the referents'. Clearly, this imposes increasing cognitive capabilities with increasing group size. He states that due to cognitive limitations, familiarity-based recognition is constrained by referent number and heterogeneity (label variability) during familiarization. We agree that under these assumptions, one should take care of group size and heterogeneity in experiments aiming to test this model of social recognition. However, there are alternative models. For example, ants groom, feed, or contact nest mates and thereby contaminate them with their own blend of cuticular hydrocarbons (Lenoir et al. 2001; Bos and d'Ettorre 2012). Ultimately, all nest mates harbour the same mix of hydrocarbons that is perceived as a single label. This is the model of the so-called Gestalt colony odour (Crozier and Dix 1979). Under this assumption, increasing group size does not pose a challenge to the cognitive capacities of group members. For the case of thrips, we do not know the cue or the cues they use to familiarize, or whether they originate from thrips individuals or from their environment. Hence, it is premature to theorize about which social recognition model applies to thrips. However, from a selectionist point of view, we expect the more simple (and less costly) social recognition system to evolve.

In addition, we emphasize that Schausberger (2014) makes two false claims as to what we concluded from our experiments (de Bruijn et al. 2014). First, he rephrases our conclusion that 'growing up together is a necessary but not sufficient condition for discrimination in thrips larvae' into 'thrips only familiarize with kin or kinship is needed for generating beneficial effects of familiarity under predation risk'. Then, he claims that this (rephrased) conclusion is invalid. However, we have never drawn this conclusion and instead found support for familiarization among thrips larvae in kin groups only. Second, Schausberger (2014) claims 'familiarity is only established, or does only have an effect, in sibling but not non-siblings' as being our conclusion. However, we recognized the difference in pre-experimental group size of siblings and non-siblings and separately analysed survival of familiar vs. unfamiliar thrips. Therefore, we carefully refrained from comparing sibling and non-sibling survival, a point that Schausberger (2014) acknowledges but subsequently ignores.

\section{References}

Bos N, d'Ettorre P (2012) Recognition of social identity in ants. Front Psychol 3:83

Crozier RH, Dix MW (1979) Analysis of two genetic models for the innate components of colony odor in social Hymenoptera. Behav Ecol Sociobiol 4:217-224

de Bruijn PJA, Sabelis MW, Egas M (2014) Effects of kinship or familiarity? Small thrips larvae experience lower predation risk only in mixed-size sibling groups. Behav Ecol Sociobiol 68:1029-1035

Lenoir A, d'Ettorre P, Errard C, Hefetz A (2001) Chemical ecology and social parasitism in ants. Annu Rev Entomol 46:573-599

Schausberger P (2014) Taking care of group size and heterogeneity in social recognition systems. Behav Ecol Sociobiol. doi:10.1007/ s00265-014-1763-2 